\title{
Prosodic anticipatory clues and reference activation in simultaneous interpretation
}

\section{[Les indices anticipatoires prosodiques et l'activation de la reference en interpretation simultanee]}

\author{
Maria Palova - Eva Kiktova
}

DOI: 10.18355/XL.2019.12.01XL.02

\begin{abstract}
Intonational patterns carry anticipation phenomena inherent to the utterers' productions. In perception, listeners learnt to exploit them with the sake to promptly and correctly retrieve the transmitted message. As far as the interpreter's performance is concerned, his/her capacity to make use of the aforementioned phenomena is of significant importance for the interpreting assignments. The tests conducted as part of our study demonstrate that it is possible to define and outline certain anticipatory prosodic strategies with a direct impact on (especially simultaneous) interpretation. They confirm that the structural information conveyed by an anticipatory clue identified by an interpreter helps to optimize his/her rendition. Multi-layered linguistic constraints of an interpreting task are thus transformed into a cognitive constraint.

The segmentation/tying function performed a posteriori retains traces of all levels of constraints as evidenced by the results of our perceptual tests.
\end{abstract}

Key words: prosody, reference, anticipatory strategies, simultaneous and consecutive interpretation, perceptual tests

\section{Résumé}

Des patterns intonatifs sont porteurs de phénomènes anticipatoires prosodiques inhérents aux productions des locuteurs. En perception, les auditeurs les ont appris à exploiter pour une récupération rapide et appropriée du message transmis. La capacité de les utiliser lors de la tâche interprétative représente un facteur important de la performance de l'interprète. Les tests réalisés dans le cadre de notre étude démontrent qu'il est possible de définir et de délimiter certaines stratégies prosodiques anticipatoires ayant un impact direct notamment sur la tâche de l'interprétation simultanée. Ils confirment que l'information structurale véhiculée par un indice anticipatoire repéré par l'interprète lui permet d'optimiser son interprétation. Dans son travail, les contraintes relevant de tous les niveaux linguistiques se transforment en contrainte cognitive. La fonction de segmentation/liage réalisée a posteriori garde les traces de tous les niveaux de contrainte comme il en témoigne les résultats de nos tests perceptifs.

Mots-clefs : prosodie, référence, stratégies anticipatoires, interprétation simultanée et consécutive, tests perceptifs

La présente étude s'inscrit dans un projet de recherche qui vise à dévoiler et à analyser des indices anticipatoires prosodiques, pour le français, exploitables lors d'une tâche d'interprétation simultanée du français vers le slovaque. Sensibilisé à ces schémas anticipatoires prosodiques, l'interprète, du français au slovaque, devrait être capable de les implémenter en prenant les décisions stratégiques convenables et rapides dans l'exécution de sa tâche d'interprétation simultanée et consécutive.

Les corpus consistent en un enregistrement de 50 heures d'une série de discours politiques en français (France) (EP live - Parlement Européen), comprenant des voix féminines (10) et masculines (10). Les données acoustiques numérisées sont 
en cours d'analyse à l'aide du logiciel Multi-Speech. L'évolution des paramètres prosodiques, suivis et extraits automatiquement (et corrigés manuellement), sont: la fréquence fondamentale ( $\mathrm{F} 0$ en $\mathrm{Hz}$ ), l'intensité (en $\mathrm{dB}$ ) et la durée (en ms et en sec), avec une attention particulière accordée à la fréquence fondamentale, afin d'étudier la trajectoire des différents patterns de l'intonation. Les durées moyennes des groupes rythmiques, courts ou longs, sont mesurées. Il en va de même des pauses remplies et silencieuses. Les vitesses d'élocution et d'articulation sont également calculées.

Prises ensemble, ces mesures nous servent à définir des contraintes phonétiques généralisables pour la construction d'un modèle conceptuel, combinant les niveaux phonétique, syntaxique et sémantique, sous forme d'un «logiciel mental» pour une interprétation optimale.

\section{Problématique}

Les phénomènes phonétiques anticipatoires sont systématiquement présents en production de la parole. L'étendue de ces stratégies anticipatoires en parole va du niveau de la microstructure, c'est-à-dire celui des segments phonétiques (voyelles, consonnes et syllabes), au niveau de la macrostructure qui concerne les mots, les phrases et un discours entier. Ainsi, en parole, les gestes articulatoires anticipatoires sont compris, au niveau de la microstructure, comme l'expansion ou l'extension de certains gestes à des segments phonétiques adjacents ou environnants. Au niveau de la macrostructure, l'extension de ces phénomènes anticipatoires dépasse le domaine des segments et des syllabes ; ils sont généralement pris en charge par des caractéristiques prosodiques, en particulier par des patterns intonatifs, et leur domaine est au moins celui du mot, mais généralement s'étalent sur de longues chaînes de phrases.

En perception de la parole, les auditeurs ont appris à exploiter ces phénomènes anticipatoires inhérents aux productions des locuteurs pour reconstruire de façon adéquate et aussi optimale que possible le message linguistique. En d'autres termes, les auditeurs utilisent des indices précoces liés à ces éléments anticipatoires qui sont présents dans la chaîne parlée pour une récupération rapide et appropriée du message que le locuteur désire transmettre.

\section{Hypothèse de recherche}

Des tests perceptifs devraient permettre de confronter les trajectoires des schémas intonatifs avec les possibilités d'anticipation des interprètes. 1) Il est fort probable que plus la courbe mélodique prendra une allure ascendante et pentue pour signaler de façon anticipatoire un référent à venir dans le discours, plus la capacité des interprètes à anticiper et à interpréter correctement sera robuste. 2) Le degré de couplage entre les trajectoires des schémas intonatifs et les possibilités d'anticipation des interprètes sera évalué aussi par un procédé d'observation de perturbations liées à l'hyperprosodie ou l'hypoprosodie (les valeurs de la fréquence fondamentale (F0) trop élevées ou trop bases, accentuation prosodique trop fréquente ou manquante). Ce paradigme expérimental devrait représenter un facteur de dégradation de la performance de l'interprète. 3) Il serait possible aussi, grâce à ces tests perceptifs, de définir et de délimiter les stratégies prosodiques anticipatoires spécifiques au locuteur, et qui auraient un impact direct notamment sur la tâche de l'interprétation simultanée.

Il est bien entendu que l'examen de la relation entre les stratégies prosodiques et l'interprétation simultanée et consécutive sera contextualisé en termes de facteurs syntaxico-sémantiques et pragmatiques inhérents au discours.

\section{Cadres théoriques}

Notre recherche prend place parmi les études qui, dans une approche sensori-motrice de la parole, tentent de démontrer l'existence d'une interaction étroite entre la perception et la production de la parole, entre le sensoriel auditif et visuel, d'une part, et le moteur (articulatoire), d'autre part. 
Ce travail s'inscrit également dans le cadre théorique de la perception auditive anticipatoire d'événements (Anticipatory Perception based of Events (APE) hypothesis). Selon ce modèle théorique, les auditeurs et les observateurs seraient capables d'exploiter des indices anticipatoires perceptibles localisés dans les signaux de la parole (Sock et al., 2011).

Nous tenons compte également de l'inventaire des fonctions assumées par la prosodie dans la communication orale présenté par Di Cristo (2013), notamment de la contribution de la prosodie à la segmentation du discours en unité hiérarchisées et à l'établissement des relations entre ces unités. En effet, les tests perceptifs réalisés lors de cette étude confirment que l'information structurale véhiculée par un indice anticipatoire repéré par l'interprète ainsi que son empan comptent parmi les plus importantes informations qui lui permettent d'optimiser son interprétation.

Notre réflexion est nourrie, pour une bonne part, par les modèles psycholinguistiques soutenant que le discours est généré de façon séquentielle permettant d'élaborer une séquence pendant que celle précédente est prononcée. De ce fait, la segmentation/liage refléterait la planification cognitive du locuteur et ferait appel à la compétence de l'auditeur/interprète de saisir son intention cognitive. Le rôle du dispositif cognitif consiste à superviser la production et la perception (Levelt, 1993). Le modèle de compétition (modèles connexionnistes) semble apporter une solution aux situations où l'hétérogénéité d'indices anticipatoires concurrents saisis par l'auditeur/interprète l'amène à choisir une interprétation parmi plusieurs possibles.

Dans le travail de l'auditeur/interprète, les contraintes relevant des niveaux syntaxique, sémantique, pragmatique, prosodique, etc. se transforment au moment des opérations de segmentation/liage en contrainte cognitive. La fonction de segmentation/liage réalisée a posteriori garde les traces de tous les nivaux de contrainte comme il en témoigne les résultats de nos tests perceptifs.

En résumant les études traductologiques portant sur la capacité de traitement individuelle de l'interprète, M. Guidère constate que « les déclencheurs de saturation chez l'interprète peuvent être classés en trois grandes catégories :

1) Le changement soudain dans le temps de traitement (ex. débit plus rapide, énumération).

2) La détérioration de la qualité du signal sonore (ex. mauvaise acoustique, discours mal accentué).

3) La mauvaise segmentation du discours ou l'apparition soudaine de segments difficiles à gérer (ex. grammaire inhabituelle, logique confuse. » (Guidere, 2011: 108 $-109)$

Nos tests perceptifs répertorient toutes les trois catégories de difficultés.

\section{Tests perceptifs}

L'anticipation est un concept reconnu notamment par les interprètes qui le caractérisent comme une suite d'indices (moments) anticipatoires qui leur permet d'interpréter correctement, sans omissions et de garder, en même temps, le contrôle de leur propre production. La forme que saisit l'oreille de l'interprète (réception) présente non seulement une morphologie mais elle contient aussi la dynamique de sa création: elle matérialise le premier niveau auquel il est possible d'attribuer un sens (perception). De ce fait, elle est une manifestation praxologique de l'anticipation. L'émergence progressive des formes (naissance et formations des formes) est un processus de microgenèse, de modulation permanente en fonction du champ thématique qui ne détermine point où le processus de sa stabilisation doit s'arrêter ; celle-ci n'est que relative et temporaire. Ce processus continu et perpétuellement inachevé de production de formes permet de comprendre que chaque forme naissante est porteuse de sens. (Rosenthal, 2004) 
Quatre auditeurs/interprètes sont soumis à l'écoute des phrases isolées, découpées du corpus décrit à l'introduction de notre article.

L'unité minimale de test est une proposition-énoncé composée d'au moins deux groupes rythmiques, terminée par le pattern intonatif conclusif, appelée phrase.

\section{Di Cristo :}

Parmi plusieurs définitions du groupe rythmique, nous choisissons celle de

«Le rythme linguistique peut être regardé comme la récurrence de groupements fondé sur une organisation hiérarchique des niveaux de proéminence syllabique (ou des niveaux d'accentuation). La production et la perception du rythme impliquent, en effet, l'émergence de groupements (ou plus précisément de groupes accentuels), que l'on appelle généralement des groupes rythmiques. D'autre part, le rythme est une structure hiérarchique, ce qui signifie qu'il participe d'une organisation formée de plusieurs niveaux relatifs de groupement, ces niveaux étant corrélés aux degrés de force de proéminences syllabiques qui contribuent à la formation de ces groupements. » (Di Cristo, 2013: 12)

La tâche des auditeurs/interprètes consiste à indiquer sur l'oscillogramme le moment de repérage d'un indice anticipatoire (donnée temporelle) et la « nature » de l'information saisie. Une analyse des résultats obtenus pour les trois cent premières phrases écoutées par quatre auditeurs/interprètes (en pré-test) nous a permis d'élaborer un répertoire d'informations anticipées liées aux données temporelles marquant l'indice anticipatoire. Le répertoire actuel, issu d'une synthèse d'informations anticipées contient actuellement 12 informations types anticipées.

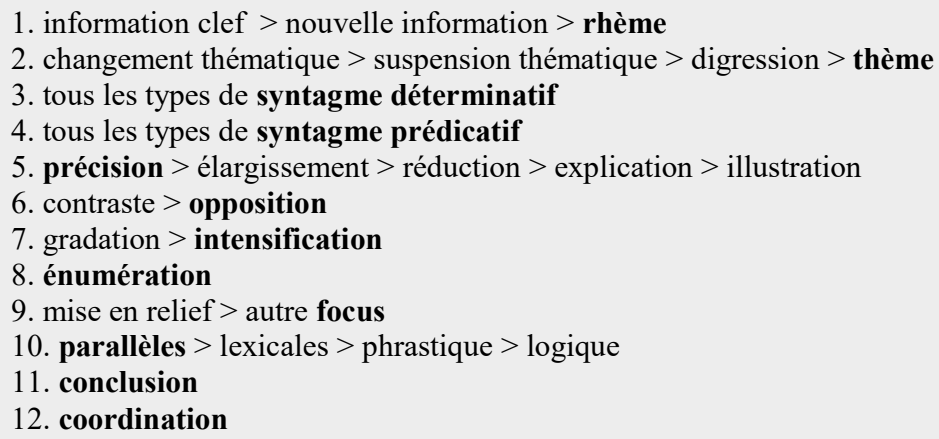

\section{Figure 1 : 12 informations types anticipées}

Cette réduction s'est montrée nécessaire et très opérationnelle lors de tests suivants réalisés au total actuel de mille phrases de corpus. Les tests ont montré que souvent à un indice anticipatoire correspondent plusieurs types d'informations anticipées et que leur configuration peut varier d'un auditeur/interprète à l'autre.

Par contre, le moment de repérage d'un indice anticipatoire sur le continuum linéaire de la phrase écoutée ne présente que très peu de variations : les quatre auditeurs/interprètes le placent sur le pic de F0 suivi d'une pause des groupes rythmiques formant des patterns intonatifs continuatifs. La plus grande quantité d'informations extraites provient des pics de F0 des deux premiers groupes rythmiques identifiés sur l'oscillogramme par les quatre auditeurs/interprètes à quelques millisecondes près (différence de rapidité d'exécution du marquage). L'empan de ces informations est long, dans certain cas cette information doit être retenue jusqu'au dernier groupe rythmique. Une variation plus importante est constatée pour les autres groupes rythmiques formant la phrase. Il arrive que les quatre auditeurs/interprètes ne s'accordent pas sur le nombre de groupes rythmiques suivants (différence produite au cours de la phase de segmentation/liage) ce qui 
explique (partiellement) la différence du nombre d'indices anticipatoires repérés par chacun d'entre eux.

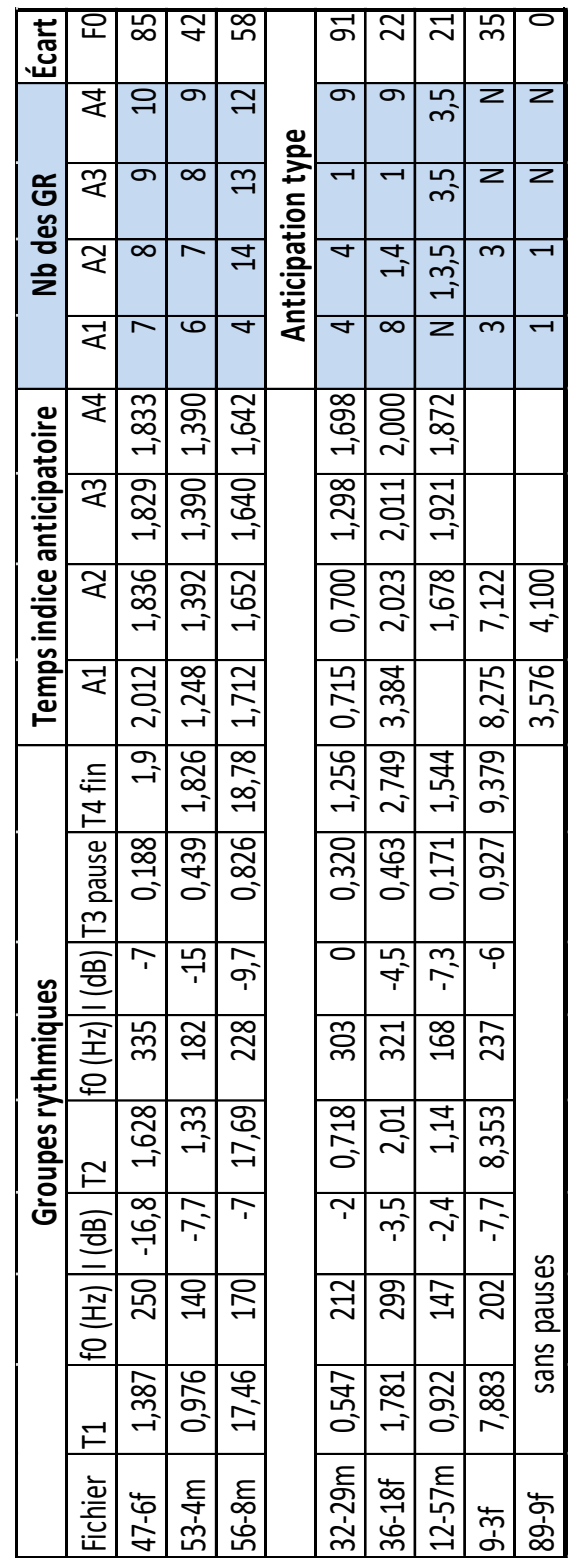

Figure 2 : Quatre perceptions d'une même phrase

Les données fournies par les auditeurs/interprètes sont dès lors soumises à l'analyse prosodique. Pour discerner la variation de F0 formant le pic d'un groupe rythmique, deux valeurs de F0 sont comparées - celles des noyaux sonnantiques de l'avant dernière et de la dernière syllabe. Ce procédé nous permet également de rendre compte de la différence entre les voix masculines et féminines étant donné que les XLinguae, Volume 12 Issue 1XL, January 2019, ISSN 1337-8384, eISSN 2453-711X 
valeurs de F0 des voix féminines sont naturellement plus hautes. Le calcul (éliminant les valeurs extrêmes mesurées) montre la valeur moyenne de l'écart de F0 = 35,98 Hz. La valeur maximale de l'écart de $\mathrm{F} 0=91 \mathrm{~Hz}$ alors que la valeur minimale de l'écart de $\mathrm{F} 0=-17 \mathrm{~Hz}$.

La pratique d'interprétation simultanée montre bien que l'interprète doit opérer un découpage en segments pertinents de l'énoncé à interpréter pour pouvoir les traiter ultérieurement. Ce découpage est fait en cours de réception et l'interprète ne traite pas consciemment chaque information anticipée et confirmée ou infirmée par la suite linéairement perçue car ce qu'il devra restituer en définitive c'est le contenu du message. D'où la difficulté des auditeurs/interprètes de se concentrer et de consciemment contrôler le « déroulement » de la perception.

Cependant, d'autres explications de la variation se proposent :

1. les indices anticipatoires ne correspondent pas toujours aux pics de F0 des groupes rythmiques,

2. certains groupes rythmiques sont identifiés mais ne livrent pas d'informations pouvant constituer un indice d'anticipation,

3. certains groupes rythmiques ne sont identifiés à cause d'irrégularités prosodiques.

Les données temporelles identifiant les indices anticipatoires indiquées sur l'oscillogramme précèdent parfois les pics de $\mathrm{F} 0$ des groupes rythmiques et l'information anticipée est le plus souvent du type 3, 5, 6 et 10 dont l'empan est court. Cependant, ces données temporelles coïncident avec les pics de F0 dont les valeurs sont plus faibles que celles des pics de F0 des groupes rythmiques. Ce constat, soutiendrait-il la distinction entre des patterns intonatifs de continuation simple et des patterns intonatifs de continuation groupante décrite par P. Delattre (1966) et C. Portes (2002)?

Dans très peu de cas, les quatre auditeurs/interprètes identifient un moment anticipatoire sur le pic de F0 d'un groupe rythmique mais l'information saisie est trop floue pour pouvoir être associée à une de nos 12 informations types (marquée dans leurs tableaux de perception comme N). Ainsi, leur mobilisation anticipatoire par le pic de F0 reste vaine.

Deux cas de figure donnent lieu à la résignation des auditeurs/interprètes d'accomplir la tâche anticipatoire lors des tests perceptifs : le premier est lié à l'éclatement prosodique de la phrase dû à l'hyperprosodie. Certaines phrases comptent jusqu'à 11-12 pics de F0 dont les valeurs sont celles des pics de F0 délimitant un groupe rythmique (valeur moyenne de l'écart de F0) ce qui oriente l'effort cognitif des auditeurs/interprètes à la segmentation à tel point que leur capacité cognitive s'en trouve saturée. Un autre type d'éclatement prosodique est observé dans les phrases où l'emphase coïncidant avec un pic de F0 délimitant un groupe rythmique (valeurs maximales de l'écart de F0) met en valeur un segment dont la pertinence n'est pas avérée par d'autres niveaux linguistiques. Là encore l'effort cognitif des auditeurs/interprètes retenu par la vérification de validité informationnelle du segment sous l'emphase mène à l'explosion informationnelle et entrave l'anticipation.

Le deuxième cas de figure représente les phrases à l'intonation plate: les valeurs des pics de F0 des patterns intonatifs continuatifs sont en dessous de la valeur minimale de l'écart de F0 ce qui produit un effet similaire au premier cas de figure où la quasi-totalité de l'effort cognitif des auditeurs/interprètes est utilisée à la segmentation. 


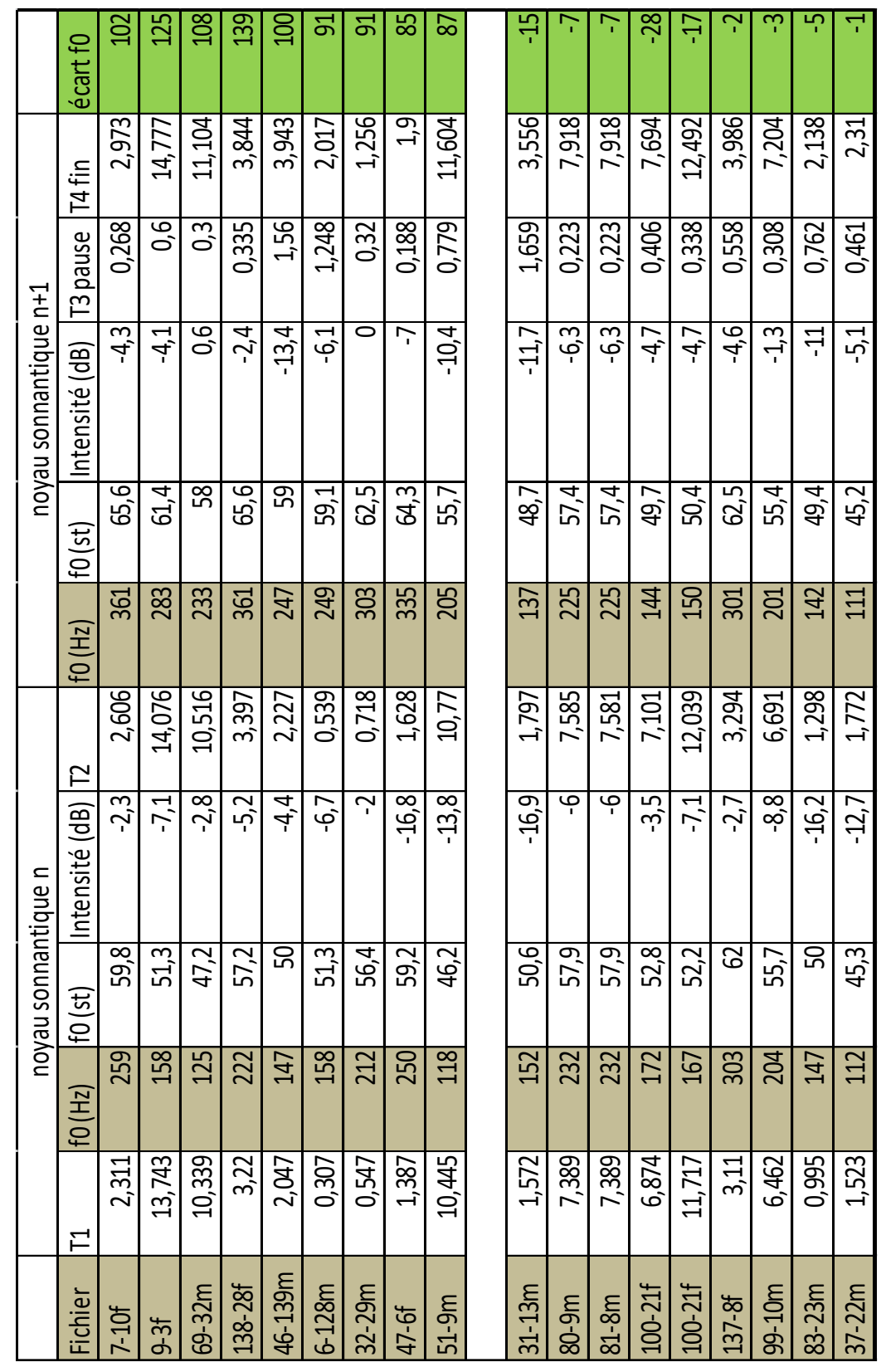

Figure 3 : Ecart entre les valeurs de F0 de deux dernières syllabes formant les pics des groupes rythmiques hyper/hypo prosodiques 


\section{Résultats}

Notre corpus est constitué de paroles produites par lecture-improvisation. L'interprétation simultanée traite surtout ce type de discours, il importe alors d'observer dans quel type d'informations elle trouve son appui. Les résultats des tests perceptifs font ressortir que l'anticipation trouve ses ressources sur tous les niveaux linguistiques ainsi que dans la connaissance et compétence thématique, discursive et communicative des quatre auditeurs/interprètes. Lors du pré-test, soumis à la tâche de décrire l'information liée à l'indice anticipatoire repéré, ils ne disposaient d'aucune règle de description de sa qualité. Pourtant, les informations décrites comme permettant d'anticiper la suite de la phrase étaient surtout celles qui portaient à sa structure (segmentation en unité hiérarchisées) : suivra une nouvelle information / un changement thématique / une énumération, etc. (cf. Fig. 1). Nous pouvons constater que les indices anticipatoires permettent :

- d'identifier les constituants syntaxiques de haut rang et de les mettre en relation,

- d'opérer la distinction fond/focus,

- de mobiliser les procédés de décodage ou d'inférence (extraire une information implicite);

- d'identifier si l'information est conceptuelle ou procédurale,

- d'opérer une distinction entre le sens informatif et le sens communicatif (reconnaitre la signification affective et le point du vue du locuteur).

Il en découle que le sens qu'un pattern intonatif aide à véhiculer dépend de l'interaction que le sens porté par la configuration du pattern intonatif entretient avec le sens porté par le matériau verbal.

Les tests confirment notre hypothèse 1) mais la capacité des interprètes à anticiper et à interpréter correctement n'est vraiment robuste qu'après les deux premiers pics de F0 des groupes rythmiques. Les indices anticipatoires repérés sur les suivants pics de F0 des groupes rythmiques sont plus menus en informations ou bien ils ne dégagent qu'une simple attente qu'il est impossible de mieux cerner. Néanmoins, l'ensemble des informations dégagées à partir des deux premiers groupes rythmiques permet de contrôler le processus de compréhension-interprétation et facilite $1^{\prime} / 1$ es ajustement(s) progressifs(s) de la référence.

Les tests confirment notre hypothèse 2). Le degré de couplage entre les trajectoires des schémas intonatifs et les possibilités d'anticipation des interprètes se trouve perturbée par le phénomène d'hyperprosodie ou d'hypoprosodie et cela dans une telle mesure que les interprètes perdent leur contrôle sur la compréhensioninterprétation (lors de la phase de la reformulation-réexpression ils ne font que glisser à la surface de mots).

Les tests confirment partiellement notre hypothèse 3 ) car ils montrent qu'il est possible de sélectionner certains patterns intonatifs où l'information type ou leur combinaison est à chaque fois définie par les quatre auditeurs/interprètes de façon univoque: il s'agit notamment du pattern intonatif introduisant le rhème, le syntagme déterminatif, syntagme prédicatif et l'énumération. Les tests montrent qu'il existe aussi leurs combinaisons spécifiques dont l'empan est suffisamment long pour une reconstruction optimale de la phrase à interpréter. L'ouïe des interprètes non natifs les reconnait sans ambigüité. Il est à supposer comme possible de choisir les patterns intonatifs associés régulièrement à une/plusieurs information(s) et d'y entrainer l'ouïe des interprètes par son exposition répétée à des phrases représentatives de ces patterns intonatifs.

\section{Discussion}

A cette étape de recherche, notre étude ne représente qu'un «premier attouchement» à la problématique des qualités prosodiques soutenant les indices anticipatoires. Comme le montre le tableau de la Figure 3, d'autres paramètres prosodiques entrent dans la 
structuration informationnelle des propositions-énoncés, notamment la longueur des pauses (la durée moyenne de la pause étant de $0,39 \mathrm{~s}$, la durée minimale de $0,03 \mathrm{~s}$ et celle maximale de $0,89 \mathrm{~s}$ ) et le débit. L'expérience des interprètes confirme que les segments prononcés à un débit plus rapide correspondent aux informations anticipées types 3, 5, 6 et 10 (segments d'incision d'une précision, d'une détermination ou d d'un parallèle) alors que les informations anticipées types 1,2, 4, 9 et 11 sont prononcées à un débit standard et suivie d'une pause conséquente permettant aux interprètes de mieux les évaluer et retenir. Le contraste crée par la formation débit + pause (segments rapides vs segments ralentis) contribue à signaler l'importance du contenu informationnel des segments. Néanmoins, ce contraste, même s'il est assez régulier, ne peut pas guider les interprètes à lui seul : il arrive que les segments à haute valeur informationnelle (informations anticipées types 1 et 2) longs peuvent être prononcés rapidement. Ainsi, cet interface prosodie I information donne à réfléchir sur le calcul des représentations prosodiques.

La perception sert d'intermédiaire de reconstitution du message à partir des indices transmis par l'onde acoustique ; elle permet d'identifier le pattern intonatif et d'examiner sa contribution à la formation progressive du sens. Les indices anticipatoires repérés aident alors à délinéariser le continuum prosodique et à le structurer. Toutefois, la réalisation des patterns intonatifs dispose davantage de degrés de liberté ce qui permet de recourir à des stratégies complexes mettant en œuvre de façon conjointe ou complémentaire plusieurs paramètres prosodiques et par le locuteur et par l'auditeur/interprète. De plus, les informations prosodiques sont polysémiques et permettent de véhiculer les informations linguistiques, paralinguistiques et extralinguistiques, toutes utilisées lors de l'interprétation. Les structures prosodiques mentales servent de cadre général de la planification phonologique et phonétique de la réalisation des contenus thématiques et la continuité naturelle de la modulation prosodique assiste le traitement perceptif de l'information : elle navigue l'attention de l'auditeur/interprète vers les segments chargés d'informations pertinentes, aide à désambiguïser (agit comme révélateur de la structure sous-jacente) et préserve l'intelligibilité de la parole produite.

\section{Remerciements}

«Le présent travail est soutenu par l'Agence slovaque de recherche et de développement conformément au Contrat $n^{\circ}$ APVV-15-0307.»

\section{Bibliographic references}

DELATTRE, P. 1966. La leçon d'intonation de Simone de Bouvoir. In Delattre (1966). Studies in French and Comparative Phonetics. Mouton \&Co. La Hague, pp. 75-82.

DI CRISTO, A. 2013. La prosodie de la parole. De Boeck, Bruxelles.

GUIDERE, M. 2011. Introduction à la traductologie. Penser la traduction : hier, aujourd'hui, demain. De Boeck, Bruxelles.

KLEIBER, G. - SOCK, R. 2006. Ce + N + Relative : Sémantique et Prosodie. Lingvisticae Investigationes vol. 29, n. 2, John Benjamins Publishing Company, pp. 251-273.

LEVELT, W. J. M. 1993. Speaking, from Intention to Articulation. MIT Press, Cambridge.

PORTES, C. 2002. Approche instrumentale et cognitive de la prosodie du discours en français. Travaux interdisciplinaires du Laboratoire Parole et Langage d'Aix-enProvence (TIPA), Laboratoire Parole et Langage, vol. 21, pp. 101-119.

ROSENTHAL, V. 2004. Perception comme anticiapation : vie perceptive et microgenèse. In: Sock R., Vaxelaire B. (Eds) : L'anticipation à l'horisont du présent. Hayen: Pierre Mardaga éditeur, 2004, pp. 13-32. 
SOCK, R. et al. 2011. Anticipatory Perception based of Events (APE) hypothesis. International Seminar on Speech Production 2011, Jun 2011, Montreal, Canada.

SOCK, R. - VAXELAIRE, B. 2004. Le diable cognitif dans les détails sensorimoteurs anticipatoires. In : L'Anticipation à l'horizon du présent. SOCK R. \& VAXELAIRE B. (Eds.), Psychologie et Sciences Humaines, Mardaga, Liège, pp. 141157.

Words: 3822

Characters: 27039 (15,02 standard pages)

PhDr. Maria Palova, PhD.

Language Information and Communication Laboratory (LICOLAB)

Department of British and American Studies

Faculty of Arts

Pavol Jozef Safarik University in Kosice

Srobarova 2, 04180 Kosice

Slovakia

maria.palova@upjs.sk

Ing. Eva Kiktova, PhD.

Language Information and Communication Laboratory (LICOLAB)

Department of Slovak Studies, Slavonic Philologies, and Communication

Faculty of Arts

Pavol Jozef Safarik University in Kosice

Srobarova 2, 04180 Kosice

Slovakia

eva.kiktova@upjs.sk 